\title{
Time-of-Flight Range Image Measurement in the Presence of Transverse Motion using the Kalman Filter
}

\author{
Lee Streeter, Member, IEEE
}

\begin{abstract}
Time-of-flight range imaging cameras measure distance to objects in their field of view, but are prone to error when objects move. At least three raw frames are required to obtain one range image, and the standard method is to read out raw frames into separate sets and process to find one range image per set. Motion during the acquisition of a set causes error in the corresponding range image. In this work the problem of motion is addressed by regarding the raw data from each pixel as a noisy time series, and using the Kalman filter to efficiently perform time-series analysis. The proposed method adapts to the effects of transverse motion, measuring a sharp range image at each raw frame. The error in the proposed method is less than the traditional approach in $\mathbf{8 0 \%}$ of tests, with no detected increase in the STD due to noise. In qualitative experimental results the visible blur is reduced.
\end{abstract}

Index Terms - Time-of-flight range imaging, optical distance measurement, transverse motion, error correction, Kalman filter.

\section{INTRODUCTION}

Time-of-flight (ToF) range image cameras measure distance from a monocular vantage point, and are are gaining popularity as a depth imaging solution in numerous applications [1], [2], [3]. ToF cameras capture three or more raw frames over time, and compute the distance measurement from all the raw frames. Since the frames are captured over time, objects in the scene are assumed to be still. This is called the static scene assumption. If objects move then the static scene assumption is violated causing error in the distance measurements. A key form of motion that causes large errors is transverse motion, i.e. motion orthogonal to the radial axis in spherical coordinates. Transverse motion becomes especially important when the edge of an object passes through the field of view of a pixel, changing both the camera-object distance and the brightness of the reflected light. This work is concerned with circumventing the static scene assumption for transverse motion by seeking a measurement of the range measurement at each raw frame.

Existing work on ToF imaging in the presence of transverse motion broadly falls into two approaches: estimation and correction of motion; and reduction of the number of raw frames and total integration time. Early work uses optical flow to estimate transverse motion and warp the raw frames to correct the motion error [4]. In later work, optical flow is combined with coded exposure to also reduce blur artifacts within each raw frame [5]. Optical flow is computationally expensive, and fast implementation requires parallel hardware that could otherwise be used for

L. Streeter is with the School of Engineering, The University of Waikato, Hamilton, New Zealand. Email: streeter@waikato.ac.nz other tasks. The motion correction process is fast if the motion is restricted to one direction that is known ahead of time [3].

Computing the distance from fewer raw frames gives less opportunity for motion to cause error. When an object edge moves with transverse motion past a pixel's field of view then some of the raw frames are of a foreground object and the rest of a background object. The data in the raw frames will be consistent for each object, and not between the two objects. The raw frames are examined at each pixel for inconsistencies in the data caused by transverse motion [6], [7], and the range measurement is calculated from the remaining, consistent, data. The reduction of used frames is more efficient than using optical flow, albeit at the cost of using fewer photons to produce the distance measurement.

The number of raw frames and the integration time can be reduced simultaneously. Leveraging the capabilities for fine pixel control of the PMD sensor (PMD Technologies, Siegen, Germany), single shot one-phase ToF imaging is performed [2]. Normal camera operation takes two simultaneous raw measurements on each pixel and subtracts one from the other [2], [8]. This subtraction greatly reduces the background light signal. Instead of subtracting, the onephase method reads out each simultaneous pair for processing. It also assumes that ambient light is adequately mitigated by placing the camera in an optically black box.

When an object moves towards or away from the camera a pixel might view the same segment of the scene during the capture of the raw frames. This is radial motion, which up to changes around the edge of the object, causes error in the form of a Doppler shift [9]. Early modeling led to a reduction of that error by appropriate processing [4], and by modification of the raw frame acquisition process [10]. In more recent work radial motion is measured, e.g using heterodyne modulation [11], continuous wave homodyne [9], and analysis of the raw data using principles from quadrature modulation and stochastic calculus [12].

The Kalman filter is a statistical analysis method for noisy time series data [13], [14]. The Kalman filter is a closed form solution to linear stochastic filtering, stochastic filtering being the general problem of inferring underlying information from stochastic processes [15]. An attractive feature of the Kalman filter is its adaptive nature, stemming from its basis in a statistical model of the change of the underlying information to be inferred. Large change increases the weight the Kalman filter puts on physical measurements, updating the model accordingly. Conversely, if the effect of random noise is larger than effects arising 
from underlying change, then the filter places more weight on the model than the data. The Kalman filter has been applied to object tracking on the raw frames of ToF camera [16], but not to the more fundamental problem of range measurement itself.

A more general solution to stochastic filtering is particle filtering [17], a Monte Carlo method involving the computationally intensive statistical simulation of distributions defined by the time series model. Recent particle filter based methods: incorporate finite impulse response filtering [18], [19]; and provide a closed form solution to the statistical problem, shifting much of the computational work to an iterative solver [20].

In this work the data from ToF cameras is treated as a noisy time series. The ToF model is transformed into an exact linearized form to ease the theoretical development. Statistical processing in the form of the Kalman filter is investigated to tackle the transverse motion problem between raw frames, as they emerge from the camera, with focus on the error due to transverse motion. Emphasis is placed on the need for an efficient solution, hence the choice of the Kalman filter. The novelty of this work is in the application of noisy time-series analysis directly to raw ToF data to address transverse motion, measuring distance at each raw frame without modification of the data capture procedure or special scene preparation. Radial motion is not addressed explicitly in this work, however, the methodology is developed with radial motion in mind for later incorporation. In Sec. II the operational theory behind ToF range imaging is briefly reviewed, and then extended to address transverse motion. In Sec. III the experimental methods are described, and in Sec. IV we give results and discuss the findings.

\section{TheORY}

Amplitude modulated continuous wave (AMCW) ToF imaging fits into the same theoretical context as other ranging techniques like interferometry [21], and radar [9]. Each of these techniques share a common principle in that electromagnetic radiation is modulated to encode the distance in the angle of the modulation waveform, either as the phase shift of a continuous wave or pulse, or the continuous wave frequency. The radiation return is processed and sampled, and the distance measurement extracted by computational means.

To measure distance, an AMCW ToF camera modulates the amplitude of a LED or laser diode using a periodic signal of frequency $f$, typically from $10 \mathrm{MHz}$ up to $150 \mathrm{MHz}$. The light return is modeled by [1]

$$
r(t)=a \sin (2 \pi f t+\phi)+b,
$$

where $a$ and $b$ are respectively the amplitude and DC components of the light return, and

$$
\phi=\frac{4 \pi f d}{c}
$$

is the phase shift induced by the travel, $c$ is the speed of light in air. Therefore, given an estimate of the phase shift $\phi$ due to the ToF of the light, it is straightforward to find $d$. The sensor is shuttered according to the demodulation signal

$$
s(t)=\frac{1}{2} \sin (2 \pi f t-\theta)+\frac{1}{2},
$$

which is in homodyne with the light source and with phase offset by $\theta$. The sensor shuttering mixes the return with the demodulation signal, and the mixed signal is integrated viz.,

$$
I(\theta)=\int_{t=0}^{T} r(t) s(t) d t \approx \frac{T a}{4} \cos (\phi+\theta)+\frac{T b}{2},
$$

which is simplified to

$$
I(\theta)=\alpha \cos (\phi+\theta)+\beta,
$$

where $\alpha=T a / 4$ and $\beta=T b / 2$. Eqn. 5 is called the correlation wavefunction, and is the fundamental equation in ToF range imaging.

The classical procedure to measure $\phi$ is to step $\theta$ evenly over $[0,2 \pi)$ to $N$ distinct values called phase steps, or phase shifts, and at each phase step measure

$$
I_{n}=\alpha \cos \left(\phi+\theta_{n}\right)+\beta .
$$

The images of $I_{n}$ values are called raw frames. The Discrete Fourier Transform (DFT) of the $I_{n}$ over $n$ is taken and $\phi$ found from the phase of the first Fourier bin.

In the remainder of the present section, the ToF theory is progressively developed to increase adaptability to dynamic scenes. A running ToF range imaging method is developed that partially extends the classical approach, just presented, to measure distance over time. Then, statistical principles are invoked and the Kalman filter adapted to the problem of measuring distance by ToF imaging of dynamic scenes.

\section{A. Running ToF Range Imaging}

A practical and flexible alternative to the DFT for estimation is to apply trigonometric expansion to Eqn. 6 and factor into the matrix product as follows [22]

$$
\begin{aligned}
& I_{n}=\alpha\left(\cos (\phi) \cos \left(\theta_{n}\right)-\sin (\phi) \sin \left(\theta_{n}\right)\right)+\beta \\
& =\left[\begin{array}{lll}
\cos \left(\theta_{n}\right) & -\sin \left(\theta_{n}\right) & 1
\end{array}\right]\left[\begin{array}{c}
\alpha_{n} \cos \left(\phi_{n}\right) \\
\alpha_{n} \sin \left(\phi_{n}\right) \\
\beta_{n}
\end{array}\right]=H_{n} X_{n},
\end{aligned}
$$

where $H_{n}=\left[\begin{array}{lll}\cos \left(\theta_{n}\right) & -\sin \left(\theta_{n}\right) & 1\end{array}\right], \quad X_{n}=$ $\left[\begin{array}{lll}\alpha_{n} \cos \left(\phi_{n}\right) & \alpha_{n} \sin \left(\phi_{n}\right) & \beta_{n}\end{array}\right]^{T}$, and.$^{T}$ is the matrix transpose. The expansion and factorization in Eqn. 7 linearizes the classical ToF model whilst maintaining exact equivalence.

Taking measurements for any three or more consecutive values of $\theta_{n}, n \in\{j, \ldots j+J\}, \quad J \geq 3$, and assuming that $X_{n}$ remains constant during the measurements over those measurements, we construct the linear system,

$$
\left[\begin{array}{c}
I_{j} \\
\vdots \\
I_{j+J}
\end{array}\right]=\left[\begin{array}{c}
H_{j} \\
\vdots \\
H_{j+J}
\end{array}\right] X_{j}^{T},
$$


Upon solving Eqn. 8 for $X_{j}$, finding $\alpha$ and $\phi$ is straightforward. Therefore, by stepping $j$, consecutive dynamic measurements of $X_{j}$ are estimated up to the averaging over each set of three raw frames. This averaging caused by inverting Eqn. 8 provides a mathematical description of interframe motion blur in ToF range imaging.

\section{B. Kalman Filter ToF Range Imaging}

Returning to Eqn. 7, the $I_{n}$ represent the flow of information built up by consecutive raw measurements. If we make the assumption of a static scene then the best estimate of $x$ at the next measurement is $X_{n+1}=X_{n}$. Including noise, we therefore have the discrete stochastic processes

$$
\begin{aligned}
X_{n+1} & =X_{n}+\varepsilon_{n}, \\
I_{n+1} & =H_{n+1} X_{n+1}+\gamma_{n},
\end{aligned}
$$

where $\varepsilon_{n}$ and $\gamma_{n}$ are samples from some distribution, which we approximate as Gaussian, with mean zero and variance specified below. Eqn. 9 is recognizably the model for the discrete linear Kalman filter [13], [14].

The Kalman filter applied to ToF camera data tests the static scene assumption by taking the difference between the predicted value of $I_{n+1}$ and the measured value from the camera. Let $Q_{n}$ be the covariance matrix of $\epsilon_{n}$, and $r_{n}$ be the variance of $\gamma_{n}$. The Kalman filter specialized to the discrete stochastic process described in Eqn. 9 follows the following steps. Given a new measurement $I_{n+1}$ with known measurement parameter matrix $H_{n+1}$ the Kalman gain is

$$
K_{n+1}=P_{n} H_{n+1}^{T} \frac{1}{H_{n+1}\left(P_{n}+Q_{n}\right) H_{n+1}^{T}+r_{n+1}},
$$

from which the updated a posteriori state estimate is given by

$$
X_{n+1}=X_{n}+K_{n+1}\left(I_{n+1}-H_{n+1} X_{n}\right),
$$

and the a posteriori estimate of covariance is

$$
P_{n+1}=\left(I_{d}-K_{n+1} H_{n+1}\right) P_{n},
$$

where $I_{d}$ is the $3 \times 3$ identity matrix.

Despite the adaptive nature of the Kalman filter, it is not guaranteed that the model of $X_{n}$ will readily adapt to discontinuities due to the edge of an object from just one new measurement. From Eqn. 9, at each pixel we make a one dimensional observation, $I_{n+1}$, of a process that that has three unknown variables in $X_{n+1}$, so from elementary linear algebra at least three raw measurements are required to measure range. The statistical nature of the Kalman filter gives rise to a naturally Bayesian viewpoint, upon which we can enforce constraints on the Kalman filter to reduce the number of raw frames required to measure distance. Specifically, one reasons a priori about $Q_{n}$ and $r_{n}$ by approximating $Q_{n}=Q$ and $r_{n}=r$ (i.e. set them independent of $n$ ), and in choosing $Q$ and $r$, enforce the constraints based on existing knowledge of the camera operation. The first constraint is the approximation of independence, namely that $Q$ is a diagonal matrix. The second constraint comes from the fact that, in hardware, the pixels are designed to perform differential measurement of the integration intensity, see e.g. [2], [8]. This differential operation minimizes $\beta$, but does not necessarily eliminate it altogether. Accordingly we set the corresponding entry in $Q$ to a small, but nonzero, value. The impact of assuming small variation in $\beta$ is a soft constraint that permits only small changes of $\beta_{n}$ with $n$. Consequently, when $\alpha$ and $\phi$ undergo large change, but $\beta$ does not, then the number of new measurements necessary to adapt to this change reduces from three to two.

Finally we need a method to address the lag, now only two frames, in measuring distance as an object moves transversely past a pixel on the camera sensor. If a flat, or near flat, object passes over a pixel's field of view for the raw frames, then an accurate measurement of distance is found. When the object leaves that pixel's field of view, an adequate number of new raw frames (at least two) are required before the distance measurement is no longer in error. Recall that the data arrives from the camera in sets of $N$ raw frames for processing. To solve this error, we first run the Kalman filter as above over the $n \in 1, \ldots, N$ raw frames. Then we reapply the Kalman filter in reverse order starting from frame $N$ and ending at frame 1. For each direction, the prediction error, $e$, of the Kalman filter is computed, where

$$
e_{n+1}=\left|I_{n+1}-H_{n+1}\left(X_{n}+K_{n+1}\left(I_{n+1}-H_{n+1} X_{n}\right)\right)\right| .
$$

The distance measurement with the smaller prediction error is chosen for the final range image output. The overall procedure is termed bidirectional Kalman filter (BKF) [23], [24]. BKF is performed in a running manner. Estimating $X_{n}$ at each phase step in each direction both require initialization of $X_{n}$. These initializations are obtained from the forward pass on the previous set, and the reverse pass on the next set.

The prediction errors are computed at each phase step and at each pixel, for both forward and reverse passes, producing images of error. A two-dimensional Gaussian image filter [25] of the prediction error images is performed before the final range image output construction to reduce spurious errors due to random noise and emphasize prediction error at the edge of moving objects.

\section{Materials and Methods}

We test the range measurement with Kalman filtering using a proprietary prototype ToF camera operating at $70 \mathrm{MHz}$ modulation frequency. Data processing is performed using custom software written in Julia v0.5.1 and executed on a 2014 Macbook Pro running Ubuntu 16.04 LTS. Three-phase-step operation is used, i.e. $\theta \in$ $\{0,2 \pi / 3,2 \pi 2 / 3\}$, and three raw frame sets are taken in quick succession totaling nine raw frames. The first threephase-step set is used to prime the processing pipeline for the forward pass of the Kalman filter, and the last set for the reverse pass. The middle set is tested for range image measurement quality. In all experiments the raw frame 
data are scaled to the range $[0-1]$ and the parameters are initialized to

$$
P_{0}=I_{d}, \quad Q=\left[\begin{array}{ccc}
0.5 & 0 & 0 \\
0 & 0.5 & 0 \\
0 & 0 & 0.01
\end{array}\right], \quad r=0.1 .
$$

In a qualitative experiment a hand and a board are waved vigorously in front of the camera in a transverse manner approximately $.6 \mathrm{~m}$ from the camera for the hand, and $1 \mathrm{~m}$ from the camera for the board. The range measurement is performed by running ToF and the BKF procedure described above, and the range images examined for visual quality features in the range images.

Two quantitative experiments are performed. The first quantitative experiment described here tests the quality of the range measurement when there is a sharp change in distance during the data collection period. A white board is affixed to a Macron 6 translation stage (Macron Dynamics, Croydon, PA, USA) and moved from $1 \mathrm{~m}$ to $3.2 \mathrm{~m}$ in $1 \mathrm{~cm}$ steps. A single pixel is chosen in the center of the board. In software, sets are randomly chosen from two different board position, and the the raw frames are loaded for both. Artificial sets are constructed where the board is virtually moved between the first and second raw frame of the middle set. Reference phase measurements are found from all nine raw frames of both positions of each pair, against which BKF and running ToF distance measurements are compared. This process is repeated 10000 times and the mean absolute error (MAE) obtained. A one sided Z-test of the proportion of instances that the BKF MAE is less than the running ToF MAE is performed. The null hypothesis is the proportion is $50 \%$, i.e. there is no difference in error, and the alternative hypothesis is that the BKF has smaller MAE than running ToF in more than $50 \%$ of examples.

The first quantitative experiment is repeated using other Kalman filter variants. Each filter is selected for its desirable properties, which we list. The Kalman-Lévy filter [26] is the extension of the Kalman filter to Lévy processes, explicitly accounting for heavy-tail distributed noise. The Kalman-Lévy filter is tested in the bidirectional approach proposed above, which we call the bidirectional KalmanLévy filter (BKLF). The BKLF has an additional parameter $\mu$, where $\mu=2$ corresponds to Gaussian noise (the Kalman filter) and $\mu=1$ a Cauchy distribution [26]. The Rauch-Tung-Striebel (RTS) [27] filter is a two pass filter that optimizes the (Gaussian) statistical model on the second, backwards, pass. The noncausal filter [28] has two useful properties: it anticipates the process one step ahead; and it makes no assumptions about the distribution of the noise. The noncausal filter is tested in one pass mode and the bidirectional approach herein. Finally, the deadbeat dissapative filter is tested [20].

In the second quantitative experiment we test the random noise level of the Kalman filter applied to the ToF data and compare to traditional three-phase-step range imaging on a static scene, and test the assumption of normality made by the Kalman filter. The white board on the

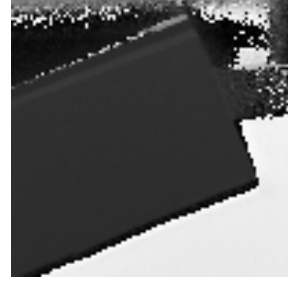

(a)

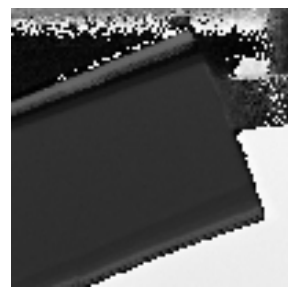

(d)

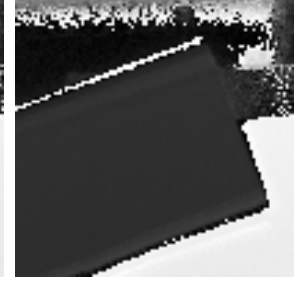

(b)

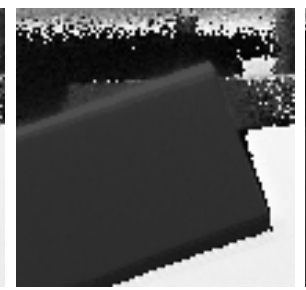

(e)

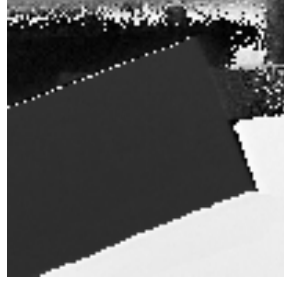

(c)

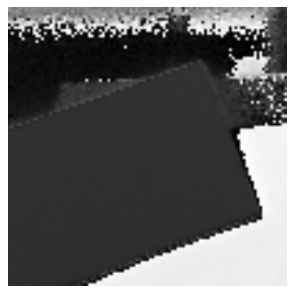

(f)
Fig. 1. Range images for the board sequence at the first raw frame (1(a) $-1(\mathrm{c}))$ and third raw frame $(1(\mathrm{~d})-1(\mathrm{f}))$. The board is traversing near diagonally downwards and slightly towards the right. The range image estimates for both the forward pass (1(a) and 1(d)) and the reverse pass $(1(\mathrm{~b})$ and $1(\mathrm{e}))$ of the Kalman filter are shown. The combined BKF output is on the right $(1(\mathrm{c})$ and $1(\mathrm{f}))$.

translation state is positioned at $2.5 \mathrm{~m}$ from the camera. The white board is aligned with the camera such that the center of the board is in the center of the camera's field of view. One hundred sets of raw frames are acquired, and an $11 \times 11$ pixel region of interest $(\mathrm{RoI})$ of the center of the board segmented for analysis. The range images are recovered from the raw frames by both Kalman filtering and traditional range imaging. The standard deviation is found at each pixel over the one hundred range images, and the mean and standard deviation of the standard deviations found over the RoI. The normality of each of the raw $I_{n}$ and estimated $X_{n}$ are tested using the Jarque-Bera test [29]. For each of the $I_{n}$ and $X_{n}, 2000$ samples are randomly selected from the RoI over the one hundred sets and tested.

\section{Results And Discussion}

In Figs. 1 and 2 the BKF progress and final range measurement images are displayed for the first and third phase steps. The multi-frame error around the leading and trailing edges of the board are apparent in the forward and reverse passes of the Kalman filter. This multi-frame error is mitigated by the BKF which takes into account the prediction error of the Kalman filter. Some edge effects remain due to intra-frame blur. The final reconstruction shows clear obscuration and revelation of the background objects, and sharp edges about the moving foreground object. Most notably, the trailing edge of the board reveals the top left corner of the background object.

In Fig. 3 we display, side-by-side, the output of running ToF and BKF at the second phase step. Notable features include, again, the motion blur of the leading and training edges of the board, and the in this frame, the small segment of background board between the top of the thumb and the 


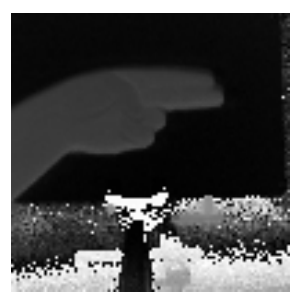

(a)

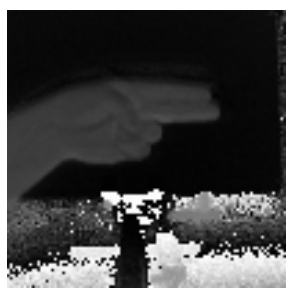

(d)

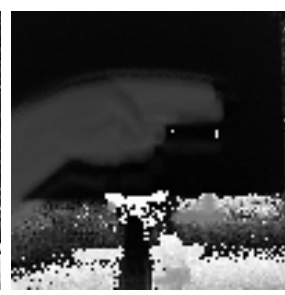

(b)

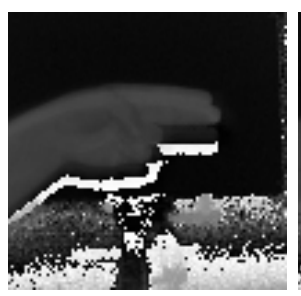

(e)

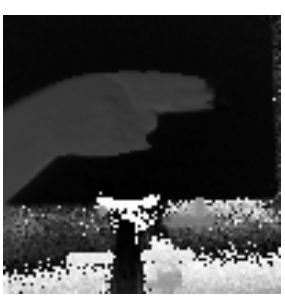

(c)

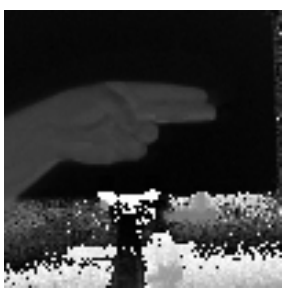

(f)
Fig. 2. Range images for the hand sequence at the first raw frame $(2(\mathrm{a})-2(\mathrm{c}))$ and third raw frame $(2(\mathrm{~d})-2(\mathrm{f}))$. The hand is traversing downwards. The range image estimates for both the forward pass (2(a) and 2(d)) and the reverse pass (2(b) and 2(e)) of the Kalman filter are shown. The combined BKF output is on the right (2(c) and $2(\mathrm{f}))$.

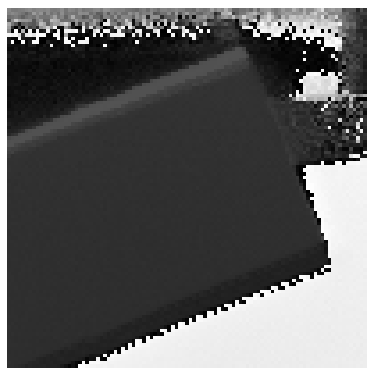

(a)

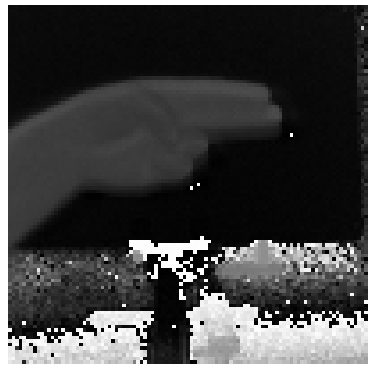

(c)

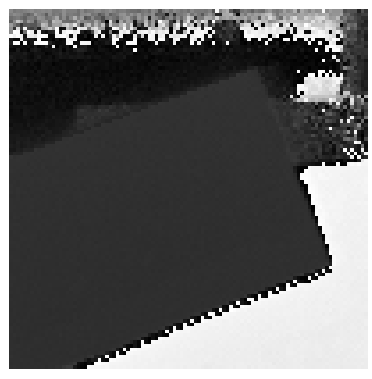

(b)

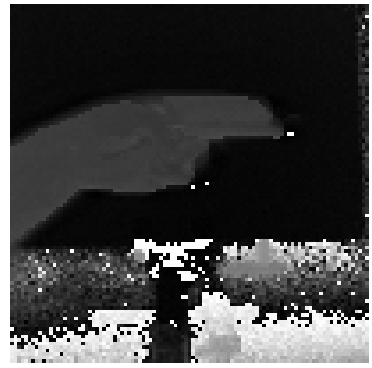

(d)
Fig. 3. Side by side comparison of running ToF range imaging (3(a) and 3(c)) with BKF (3(b) and 3(d)). All examples are the range image at the second raw frame. The edges of the BKF output are sharper than running ToF in both examples. Some edge effects are still apparent in the BKF results due to intra-frame blur.
TABLE I

Comparison of methods. The MAE is in units of Radians. BKF IS THE PROPOSED METHOD ABOVE, BKLF IS THE BIDIRECTIONAL KALMAN-LÉVy FILTER, RTS IS THE TWO PASS Smoother, and Noncaus. And B. Noncaus. are the nOnCAUSAL AND BIDIRECTIONAL NONCAUSAL NOISE-DISTRIBUTION-AGNOSTIC FILTERING METHOD.

\begin{tabular}{c|ccc} 
Data & $\%$ & $(\mathrm{Z}, \mathrm{p})$ & MAE mean $(\mathrm{STD})$ \\
\hline BKF & 80 & $(190,<0.0001)$ & $0.36(0.61)$ \\
BKLF & 82 & $(209,<0.0001)$ & $0.33(0.57)$ \\
RTS & 80 & $(190,<0.0001)$ & $0.71(0.70)$ \\
Noncaus. & 41 & NA & $0.77(0.71)$ \\
B. Noncaus. & 60 & $(63,<0.0001)$ & $0.66(0.79)$ \\
Trad & NA & NA & $0.75(0.64)$
\end{tabular}

side of the first finger visible in BKF.

The first quantitative experiment tests the range measurement by BKF and running ToF. The mean(STD) of the MAEs was $0.75(0.64)$ radians for running three-phasestep ToF and 0.36(0.61) radians for BKF. The MAE by BKF was less than three-phase-step ToF in $80 \%$ of the 10000 tests, which is significant $(Z=190, p<0.0001)$. Therefore, while BKF is detectably superior to running ToF imaging, the difference is not beyond the influence of random artifacts. Regardless, this result strengthens the qualitative result that error due to sharp scene changes is mitigated.

In Table I we give the comparison of methods for the first quantitative experiment. The optimal value for $\mu$ is found by inspection to be 1.9, representing noise that is close to Gaussian but with a slight heavy tail. BKLF narrowly outperforms BKF, but with the added computational cost of two eigen-decompositions at each time point. The RTS filter outperforms traditional three phase step ToF as often as BKF, but the MAE of RTS filtering is nearly twice as large as BKF. We deduce that RTS performance is increased by its second pass, but does not address the key issue of this work, namely step change motion error. Noncausal filtering performs worse than traditional ToF, hence the significance test is not reported, but is improved by the bidirectional approach. The deadbeat dissipative filter on three phase step ToF gave the same result as traditional ToF, so is not listed in Table I.

The second quantitative experiment probes the relative influence of noise on three-phase-step classical range measurement and BKF by examining the STD over an $11 \times 11$ RoI of a static scene. The Mean(STD) of the STD for three-phase-step ToF is 0.019(0.001) radians and for BKF is $0.019(0.001) \mathrm{rad}$. Therefore, no difference between the two methods in random noise behavior is found. The STD of the STD is low in both cases, indicating homoscedasticity over the $11 \times 11$ pixel RoI.

The second quantitative experiment also tests the normality of the raw frames and the estimated $X_{n}$ by BKF. The Jarque-Bera test is used, examining the deviation of skew and kurtosis from that of a normal distribution. The tests were performed with significance level 0.01 , reject- 
TABLE II

SIGNIFICANCE TESTS OF NORMALITY OF THE RAW DATA AND THREE ENTRies of the ESTimated $X_{n}$ AT THE Ninth Phase STEP OF A STATIC SCENE. JB IS THE TEST STATISTIC, WHERE High VAlues INDICATE NON-NORMALITY OF THE DATA.

\begin{tabular}{c|ccc} 
Data & Decision & P & JB \\
\hline Raw Step 1 & 0 & 0.23 & 2.85 \\
Raw Step 2 & 0 & 0.21 & 3.03 \\
Raw Step 3 & 0 & 0.26 & 2.65 \\
Raw Step 4 & 0 & 0.50 & 0.90 \\
Raw Step 5 & 0 & 0.07 & 5.10 \\
Raw Step 6 & 1 & $<0.01$ & 9.82 \\
Raw Step 7 & 0 & 0.09 & 4.69 \\
Raw Step 8 & 0 & 0.06 & 5.43 \\
Raw Step 9 & 1 & 0.03 & 6.86 \\
Output 1 & 0 & 0.05 & 0.13 \\
Output 2 & 0 & 0.05 & 0.94 \\
Output 3 & 1 & $<0.01$ & 17.1
\end{tabular}

ing the null hypothesis when JB $>9.70$. The results are summarized in Table II. Raw step 6 was detected as not normal, primarily due to discretization caused by the camera's digitization of data affecting the kurtosis. However, the associated JB value is close to the threshold, so the deviation from normality is moderate. We cannot reject the null hypothesis for the first and second terms of $X_{n}$, but the third term was detected as not normal with a high JB due to heavy tails. The detection of heavy tails agrees with the BKLF outperforming BKF. The exact cause of the heavy tails is currently unknown, and most pertinently future work will determine if the heavy tails are a fundamental feature common to all such ToF imaging cameras warranting the use of appropriately specialized time-series analysis techniques.

\section{Conclusion}

We have applied the Kalman filter to ToF imaging to measure distance, despite the presence of transverse motion, and at each phase step of the ToF data capture process. The Kalman filter was performed both forwards and backwards, termed the bidirectional Kalman filter (BKF). Each direction returns an estimate of distance. Recomputing the camera measurement from each BKF output, the measurement that best matched raw camera data was chosen at the correct one. Qualitative experiments demonstrated that the BKF output reduced visible error. In a quantitative experiment BKF had less error than classical ToF distance measurement in $80 \%$ of tests.

The main advantage of the proposed BKF method is measurement of distance at each raw frame, albeit at a slight increase in computational load due to the need to visit each pixel twice. All available information is treated as important and incorporated into the measurement. Moreover, the nature of statistical approaches makes BKF extensible. In future work we will subsume radial motion measurement into this transverse model, and measure the transverse motion itself.

\section{REFERENCES}

[1] D. Lefloch, R. Nair, F. Lenzen, H. Schäfer, L. Streeter, M. J. Cree, R. Koch, and A. Kolb, Technical Foundation and Calibration Methods for Time-of-Flight Cameras. Berlin, Heidelberg: Springer Berlin Heidelberg, 2013, pp. 3-24.

[2] S. Hussmann and A. Hermanski, "One-phase algorithm for continuous wave TOF machine vision applications," IEEE Transactions on Instrumentation and Measurement, vol. 62, no. 5, 2013.

[3] S. Hussmann, A. Hermanski, and T. Edeler, "Real-time motion artifact suppression in tof camera systems," IEEE Transactions on Instrumentation and Measurement, vol. 60, no. 5, pp. 1682 $1690,2011$.

[4] M. Lindner and A. Kolb, "Compensation of motion artifacts for time-of-flight cameras," in Dynamic 3D Vision Workshop, vol. 5742, Jena, Germany, September 2009, pp. 16-27.

[5] L. Streeter and A. A. Dorrington, "Coded exposure correction of transverse motion in full-field range imaging," Optical Engineering, vol. 53, no. 10, pp. 102 109-1-102 109-10, 2014.

[6] S. Lee, "Time-of-flight depth camera motion blur detection and deblurring," IEEE Signal Processing Letters, vol. 21, no. 6, pp. 663-666, 2014.

[7] D. Jimenez, D. Pizarro, and M. Mazo, "Single frame correction of motion artifacts in pmd-based time of flight cameras," Image and Vision Computing, vol. 32, no. 12, pp. 1127-1143, 2014.

[8] C. S. Bamji, P. OConnor, T. Elkhatib, S. Mehta, B. T. L. A. Prather, D. Snow, O. C. Akkaya, A. Daniel, A. D. Payne, T. Perry, M. Fenton, and V.-H. Chan, "A $0.13 \mu \mathrm{m}$ cmos systemon-chip for a 512424 time-of-flight image sensor with multifrequency photo-demodulation up to $130 \mathrm{mhz}$ and $2 \mathrm{gs} / \mathrm{s}$ ad," IEEE Journal of Solid-State Circuits, vol. 50, no. 1, pp. 303$319,2015$.

[9] R. Whyte, L. Streeter, M. J. Cree, and A. A. Dorrington, "Application of lidar techniques to time-of-flight range imaging," Applied Optics, vol. 54, no. 33, pp. 9654-9664, Nov 2015.

[10] B. M. M. Drayton and D. A. C. A. A. Dorrington, "Phase algorithms for reducing axial motion and linearity error in indirect time of flight cameras," IEEE Sensors Journal, vol. 13, no. 9, pp. 3386-3396, 2013.

[11] F. Heide, W. Heidrich, M. Hullin, and G. Wetzstein, "Doppler time-of-flight imaging," Transactions on Graphics, vol. 34, no. 4, pp. 36:2-36:11, 2015.

[12] L. Streeter, "Methods for linear radial motion estimation in time-of-flight range imaging," in Proc. SPIE Vol. 10332, Videometrics, Range Imaging, and Applications, Munich, Germany, 2017, p. To appear.

[13] R. E. Kalman, "A new approach to linear filtering and prediction problems," Journal of Basic Engineering, vol. 82, no. 1, pp. 3545,1960 .

[14] H. Madsen, Time Series Analysis. Boca Raton, Florida, USA: Capman and Hall/CRC, 2008, ch. 10 State space models of dynamic systems.

[15] F. C. Klebaner, Introduction to Stochastic Calculus with Applications, 3rd ed. London, UK: Imperial college Press, 2012.

[16] J. Stühmer, S. Nowozin, A. Fitzgibbon, R. Szeliski, T. Perry, S. Acharya, D. Cremers, and J. Shotton, "Model-based tracking at $300 \mathrm{hz}$ using raw time-of-flight observations," in 2015 IEEE International Conference on Computer Vision (ICCV), Santiago, Chile, December 2015, pp. 3577-3585.

[17] A. Doucet, N. de Fritas, and N. Gordon, Eds., Sequential Monte Caro Methods in Practice. New York, USA: Springer, 2001.

[18] J. M. Pak, C. Ahn, Y. Shmaliy, and M.Taeg, "Improving reliability of particle filter-based localization in wireless sensor networks via hybrid particle/FIR filtering," IEEE Transaction on Industrial Informatics, vol. 11, no. 5, pp. 1089-1098, 2015.

[19] J. M. Pak, C. K. Ahn, Y. S. Shmaliy, P. Shi, and M. T. Lim, "Accurate and reliable human localization using composite particle/FIR filtering," IEEE Transactions on Human-Machine Systems, vol. 47, no. 3, pp. 332-342, 2017.

[20] C. K. Ahn, P. Shi, and M. V. Basin, "Deadbeat dissipative FIR filtering," IEEE Transactions on Circuits and Systems-I: Regular Papers, vol. 63, no. 8, pp. 1210-1221, 2016.

[21] A. Kadambi, J. Schiel, and R. Raskar, "Macroscopic interferometry: Rethinking depth estimation with frequency-domain time-of-flight," in 2016 IEEE Conference on Computer Vision and Pattern Recognition (CVPR), Seattle, WA, USA, 2016, pp. 893-902. 
[22] L. Streeter, M. J. Cree, and A. A. Dorrington, "A strategy for the correction of effects of jitter in AMCW lidar images," in The 28th International Conference of Image and Vision Computing New Zealand, Wellington, New Zealand, 2013, pp. 500-505.

[23] X. Hu, D. V. Prokhorov, and D. C. Wunsch, "Time series prediction with a weighted bidirectional multi-stream extended Kalman filter," Neurocomputing, vol. 70, no. 13-15, pp. 23922399, 2007.

[24] Y. E. Goh, P. Raveendran, and Y. L. Goh, "Robust speech recognition system using bidirectional Kalman filter," IET Signal Processing, vol. 9, no. 6, pp. 491-497, 2015.

[25] R. C. Gonzalez and R. E. Woods, Digital Image Processing. Upper Saddle River, New Jersey, USA: Prentice Hall, 2002, vol. Second.

[26] D. Sornette and K. Ide, "The Kalman-Lévy filter," Physica D, vol. 151 , no. $2-4$, pp. $142-174,2001$.

[27] H. E. Rauch, F. Tung, and C. T. Striebel, "Maximum likelihood estimates of linear dynamic systems," AIAA Journal, vol. 3, no. 8, 1965

[28] G. A. Einicke, "Optimal and robust noncausal filter formulations," IEEE Transactions on Signal Processing, vol. 54, no. 3, pp. 1069-1077, 2006.

[29] C. M. Jarque and A. K. Bera, "A test for normality of observations and regression residuals," International Statistical Review, vol. 55, no. 2, pp. 163-172, 1987. 